\title{
IRON AND MICROBIAL INFECTION
}

\section{Ulrich E. Schaible and Stefan H. E. Kaufmann}

Abstract | The use of iron as a cofactor in basic metabolic pathways is essential to both pathogenic microorganisms and their hosts. It is also a pivotal component of the innate immune response through its role in the generation of toxic oxygen and nitrogen intermediates. During evolution, the shared requirement of micro- and macroorganisms for this important nutrient has shaped the pathogen-host relationship. Here, we discuss how pathogens compete with the host for iron, and also how the host uses iron to counteract this threat.

Gold is for the mistress, silver for the maid, copper for the craftsman, cunning at his trade, 'Good' said the Baron, sitting in his hall, 'but iron — cold iron is master of them all'.

Rudyard Kipling

A microbiological view of the crosstalk between pathogenic invaders and their victims primarily focuses on microbial survival strategies that are directed at compromising the immune defences of the host. However, this view often downplays the appealing ecologic niche that is provided by the host for these microorganisms and the crucial part that is played by the seclusion of pathogens from essential nutritional resources in host defence. During evolution, microbial settlers invaded distinct niches in the host ecosystem and developed several mechanisms to exploit indigenous nutritional resources, such as iron. Iron is an essential cofactor for many basic metabolic pathways, including the respiratory pathways of both the host and the microorganism. In response to infection, the host has developed countermeasures to limit the availability of iron to the pathogen. Furthermore, iron is vital to many functions of the macroorganism, including antimicrobial defence. Various intracellular bacteria, fungi and protozoa use distinct host-cell compartments as niches for survival, where access to essential nutrients, such as iron, is an important driving force in their evolution. An interesting example is the strategy used by mycobacteria, which block phagosome maturation to access cellular iron resources. Other microorganisms prefer the nutrient-rich lysosomal compartment, despite its hostile features, whereas others escape into the cytoplasm (FIG. 1). A better understanding of the distinct niches in the host that are selected by microorganisms and the nutritional needs of the different partners in these associations will provide improved guidelines for the development of nutritionally based intervention strategies to inhibit the growth of microbial pathogens. In this review, we discuss the microorganism-host relationship with respect to their competing and shared interests in iron: for growth and survival, in the case of microorganisms; for defence, in the case of the host.

\section{The host}

Iron is essential for the host and the pathogen, as both require this metal as a cofactor or as a prosthetic group for essential enzymes that are involved in many basic cellular functions and metabolic pathways. Ferric iron $\left(\mathrm{Fe}^{3+}\right)$ is almost insoluble under aerobic, aqueous and neutral $\mathrm{pH}$ conditions. As free iron catalyses the formation of free oxygen radicals through the Fenton reaction, it is also highly toxic to cells, resulting in protein denaturation, DNA breaks and lipid peroxidation. Therefore, in the host, iron is usually only available when it is bound to specific proteins, such as transferrin (TF), lactoferrin (LF) and ferritin, or when it is complexed to haem within haemoproteins ${ }^{1,2}$. Consequently, free iron is relatively unavailable in the host. The solubility of the predominant form of iron, $\mathrm{Fe}(\mathrm{OH})_{2}^{+}$, is $1.4 \times 10^{-9} \mathrm{M}$ at $\mathrm{pH} 7$, which increases to $10^{-3} \mathrm{M}$ at $\mathrm{pH} 5$ (REF. 3). Iron is taken up in the intestine either as free ionic iron - after $\mathrm{Fe}^{3+}$ reduction to ferrous iron $\left(\mathrm{Fe}^{2+}\right)$ - which is catalysed by an intestinal ferrireductase (probably duodenal cytochrome $b$ (DCYTB)) 


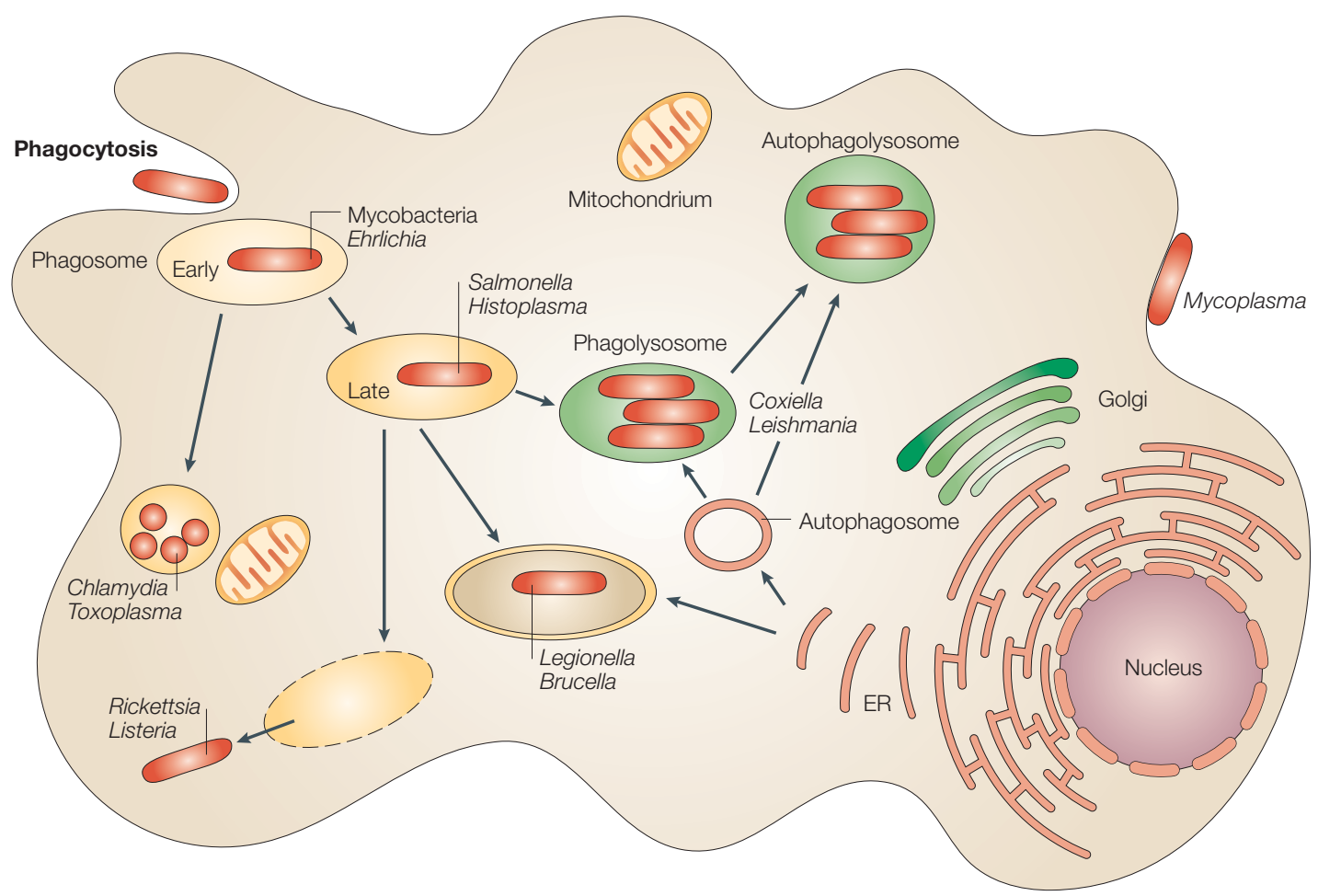

Figure 1 | The niches of host-cell-associated microorganisms. Pathogenic microorganisms occupy various niches in host cells. Whereas mycoplasmae adhere to the host-cell membrane to access nutrients from the cell, many pathogens dwell in the host cell. These intracellular pathogens either associate with distinct stages of the phagosomal-endosomal system or escape from the phagosome into the cytoplasm (for example, listeriae and rickettsiae). Mycobacteria, ehrlichiae, salmonellae and histoplasmae inhibit the maturation of phagosomes. Coxiellae and leishmaniae prefer acidic, hydrolase-rich late endosomal/lysosomal compartments, and leishmania vacuoles interact with autophagosomes - the recycling system of the host cell. Legionellae and brucellae associate with membranes of the endoplasmic reticulum (ER) and chlamydiae and toxoplasmae associate with mitochondria.

DEFENSINS

Small basic peptides produced by immune cells that mediate their microbicidal effects by damaging bacterial membranes.

\section{B-2-MICROGLOBULIN}

$(\beta 2 \mathrm{~m})$. A $12-\mathrm{kDa}$ protein that is non-covalently associated with MHC class-I molecules and their homologues - CD1 and HFE.

EARLY ENDOSOME

Early stage of an intracellular vesicle after endocytosis, characterized by the presence of the transferrin receptor and a mildly acidic $\mathrm{pH}$.
- or complexed to haem or other chelators. Reticuloendothelial macrophages recycle most iron by the phagocytosis and subsequent lysosomal degradation of old erythrocytes to release haem-bound iron. Furthermore, free haem-bound iron in the form of haemo- or haptoglobin is taken up by macrophages through the haemoglobin-scavenger receptor (CD163) (REF. 1). In the intestine, the divalent-metal transporter-1 (DMT1), a permease that is also known as DCT1, SLC11A2 or NRAMP2 (natural resistance-associated macrophage protein-2), participates in iron absorption ${ }^{4}$. The export of iron from the basolateral membrane of the enterocyte is thought to be facilitated by another permease, ferroportin (also called IREG1, MTP1, or SLC40A1) ${ }^{5}$ (FIG. 2).

Hepcidin-1 and -2 are peptides with 20-25 aminoacid residues - originally described as DEFENSIN-like antimicrobial peptides - which are trimmed from larger precursor polypeptides in the liver ${ }^{5}$. Hepcidin-1 plays an important part in regulating iron homeostasis, and is induced by dietary iron overload, as well as by lipopolysaccharides from Gram-negative bacteria. Genetic deletion of hepcidin in mice leads to iron overload (haemochromatosis), whereas transgenic overexpression causes anaemia ${ }^{6}$. Hepcidins probably limit both intestinal iron absorption and the release of iron from macrophages to avoid accumulation of any excess of iron. For circulation in the body, $\mathrm{Fe}^{2+}$ is oxidized to $\mathrm{Fe}^{3+}$ by either a membrane-associated or a plasmalocated oxidase, hephaestin or caeruloplasmin, respectively, which allows interaction with apoTF (REF. 1). Finally, cells take up iron in the iron-saturated form of TF, known as holoTF, which contains two $\mathrm{Fe}^{3+}$ ions. For receptor-mediated endocytosis, holoTF must bind to the TF receptor (TFR), a process that might also involve the haemochromatosis protein HFE, a $\beta$-2-MICROGLOBULIN $(\beta 2 \mathrm{~m})$-associated cognate of major histocompatibility complex (MHC) class-I molecules. The TF-TFR complex delivers iron to the EARLY ENDOSOME, where it is released for transport into the cytoplasm, probably through DMT1. In the cytoplasm, the iron is stored bound to ferritin ${ }^{7}$ (FIG. 2). The components apoTF and the TFR are recycled to the cell surface. Mutations in the HFE gene cause one of the most prevalent hereditary diseases in humans. About 5 in 1,000 Caucasians in the United States are homozygous for a specific mutation in the HFE gene (C282Y), although there are relatively fewer clinical cases of haemochromatosis ${ }^{8,9}$. Haemochromatosis is characterized by an overload of free iron in the tissues, prevalent free-oxygen radicals and cell damage ${ }^{9}$. Recent studies indicate that clinically silent mutations in the HFE gene might be important risk factors for developing chronic hepatitis after infection with the hepatitis $\mathrm{C}$ virus ${ }^{10}$. Although the TFR 


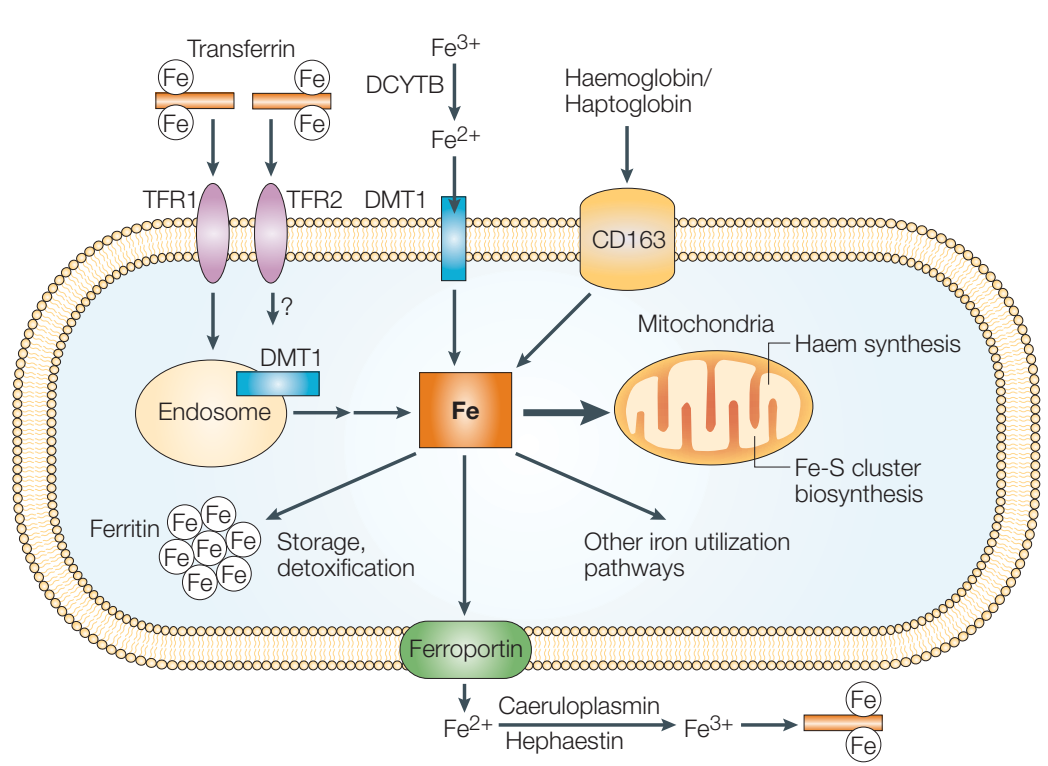

Figure 2 | Iron metabolism in the host. The iron-saturated form of transferrin, holotransferrin, binds to the transferrin receptor (TFR), which is expressed on the surface of the cell. Iron is transferred to the early endosome, where it is exported into the cytoplasm, probably through DMT1. In the intestinal lumen, ferric iron $\left(\mathrm{Fe}^{3+}\right)$ is reduced to ferrous iron $\left(\mathrm{Fe}^{2+}\right)$ by the cytochrome- $b$-like reductase DCYTB. DMT1 then facilitates the absorption of $\mathrm{Fe}^{3+}$ from the lumen into the cytoplasm. In the cytoplasm, iron is stored bound to ferritin. In macrophages, haem-bound iron - in the form of haemoglobin or haptoglobin - is taken up by the cell through the haemoglobin scavenger receptor (CD163). Iron is exported from the enterocyte by a permease known as ferroportin. For circulation in the body, $\mathrm{Fe}^{2+}$ is oxidized to $\mathrm{Fe}^{3+}$ by either the intestinal membrane-associated oxidase, hephaestin, or the plasma-located oxidase, caeruloplasmin. In the cell, the synthesis of haem and Fe-S clusters takes place in the mitochondria. Modified with permission from REF. 1 ○ (2004) Elsevier. DCYTB, duodenal cytochrome $b$; DMT1, divalent-metal transporter-1.

\section{LATE ENDOSOME}

Later stage of the endosome, characterized by the presence of hydrolytic enzymes and an acidic $\mathrm{pH}$.

\section{SIDEROPHORES}

Low-molecular-weight molecules that sequester extracellular iron for bacterial uptake.

LYME DISEASE A disease transmitted by ticks that presents with inflammation in the skin, joints heart and/or nervous system. has been shown to interact with HFE, and the crystal structure of HFE has been resolved ${ }^{11,12}$, the precise function of this protein, as well as its role in the regulation of tissue iron overload, have not been fully explained. Hepcidin plays a part in this process as the levels of this molecule in HFE-deficient mice are also low, and overexpression of hepcidin in these mice corrects haematochromatosis ${ }^{13}$. Also, other forms of haemochromatosis are caused by mutations in the genes that encode hepcidin, TFR2 (a low-affinity receptor for TF in the duodenal crypts, hepatocytes and platelets) and ferroportin ${ }^{8}$. A mutation in the ferroportin-1 gene has been associated with iron overload in Africans and African-Americans ${ }^{8}$.

Recent studies have also identified a TF-independent iron uptake system. The neutrophil-gelatinase-associated lipocalin (NGAL/24p3) complexes iron, probably through the binding of lipophilic chelators ${ }^{2}$. NGAL is secreted by epithelial cells and neutrophils and, following interaction with these iron chelators, the complex is transported to LATE ENDOSOMES. This mechanism is thought to be important in developing epithelia. NGAL can also bind ferric-enterobactin, a bacterial SIDEROPHORE. It has been suggested that NGAL-mediated siderophore capture and iron sequestration might interfere with bacterial growth and therefore have an antibacterial function. This proposal, however, requires experimental verification.
In murine macrophages, a late-endosomal membrane protein, known as NRAMP1, has a role in the innate defence response against various phagosomal pathogens, including Salmonella typhimurium, Mycobacterium bovis bacillus Calmette-Guerin (BCG) and Leishmania major ${ }^{14}$. Mice with a mutated Nrampl gene are more susceptible to infection with these organisms compared with control mice. NRAMP1 functions as a divalent-metal-ion transporter and is believed to deplete late endosomal compartments of iron by transporting it into the cytoplasm ${ }^{15}$. NRAMP1 might also be the source of iron that is required for the generation of microbicidal effector molecules, such as reactive oxygen intermediates (ROI) and reactive nitrogen intermediates (RNI). Interestingly, S. typhimurium-containing phagosomes mature to late endosomes in macrophages from resistant mice with a functional Nramp1 gene, but not in macrophages from susceptible mice with a mutated gene ${ }^{16}$. The removal of iron from these macrophages through the addition of exogenous chelators mimics the NRAMP1 resistant phenotype leading to phagosome maturation. However, iron depletion also inhibits ROI production in mice and, owing to the loss of this important anti-bacterial defence mechanism, growth of S. typhimurium is unrestrained ${ }^{17}$. Unexpectedly, although the Nramp1 gene mutation renders mice more susceptible to attenuated $M$. bovis BCG, it increases resistance to virulent Mycobacterium tuberculosis by an unknown mechanism ${ }^{18}$.

\section{The pathogen}

Iron is an important growth factor for pathogenic bacteria, with the exception of the LYME DISEASE agent, Borrelia burgdorferi, which persists in environments with very low concentrations of iron ${ }^{19}$. This is because of the selective loss of loci that encode iron-dependent proteins from the genome of B. burgdorferi. Iron concentrations of $10^{-6}$ to $10^{-7} \mathrm{M}$ are required by most microorganisms for various metabolic processes that are crucial for microbial replication, including electron transport, glycolysis, DNA synthesis and defence against toxic ROI ${ }^{20}$. Specialized iron-uptake systems have been identified in most bacterial species studied so far, and these allow microorganisms to compete for this vital element within mixed microbial communities in the environment. These systems might be pre-adaptations that evolved in the environmental ancestors of pathogenic species, thereby facilitating their colonization in a living environment, the mammalian host. In the context of iron availability, the host is an extremely hostile environment for microorganisms, which require around $10^{11}-10^{12}$-fold higher concentrations of free iron than is physiologically available ${ }^{3}$.

Specialized iron-acquiring systems in pathogenic bacteria consist of different molecules, which function in a highly interrelated mode. Iron-binding molecules, siderophores, include the mycobactins and exochelins in mycobacteria, TF-like molecules in Listeria monocytogenes and the ferric enterobactins in Gram-negative bacteria $^{3,21}$. The subsequent uptake of iron-bound ligands depends on siderophore receptors and ATP-consuming porin-like transporters in the bacterial outer membrane. Siderophores can be classified into three groups: first, 
phenolic rings with hydroxy and dihydroxybenzoic acid groups for chelation, second, hydroxamate structures with carboxy and aminohydroxy groups and third, a combination of both ${ }^{21-23}$. M. tuberculosis synthesizes siderophores of the latter two types. Mycobacteria, nocardiae and rhodococci are unique in that they synthesize a membrane-associated mycobactin, as well as extracellular siderophores known as carboxymycobactin and exochelin ${ }^{22}$. It is assumed that exochelins take up $\mathrm{Fe}^{3+}$ from the environment and subsequently bind to a receptor in the bacterial cell wall, which directs further transport through an ATP-dependent transporter (composed of FxuA, B and C; FIG. 3). It has also been proposed that, on binding $\mathrm{Fe}^{3+}$, carboxymycobactin enters the cell wall through a porin and transfers iron to a two-component metal-transport system (composed of Irp10 and Mta72) in the cell membrane. In both cases, the excess iron is probably captured by mycobactin ${ }^{22}$. Finally, $\mathrm{Fe}^{3+}$ is reduced to $\mathrm{Fe}^{2+}-$ by a process that involves reductases - for subsequent incorporation into haem- or iron-containing proteins (FIG. 3).

In Gram-negative bacteria, the receptor for ferric enterobactin, FepA, is located in the outer membrane of the organism. This receptor transfers iron to a periplasmic protein, FepB, in a TonB-dependent fashion. Transport across the cell membrane is accomplished by an ATP-dependent permease. In Escherichia coli, at least two additional iron uptake systems have been described (reviewed in REF. 3). In mycobacteria, an NRAMP homologue, known as mycobacteria (M)RAMP, is presumed to transport divalent metal ions, including iron, counteracting the activity of the host divalent-metal transporter, DMT1 (REF. 24). A periplasmic iron-binding protein, FxuD, and an inner-membrane ABC-TRANSPORTER, the Fxu complex, also promote entry of free iron into the cell where it binds to iron storage proteins including the bacterioferritins (FIG. 3). The expression of genes involved in iron uptake and storage are controlled at the level of transcription and the factors that regulate these genes are usually essential. One such transcription factor in mycobacteria is the iron-dependent regulator IdeR, a homologue of the diphtheria-toxin repressor (DtxR) in Corynebacterium diphtheriae ${ }^{25-27}$. In its $\mathrm{Fe}^{2+}$-bound state, IdeR represses genes that encode iron acquisition molecules and activates the expression of iron storage proteins and oxidative stress responses. In Gram-negative bacteria, low-molecular-weight proteins that are encoded by the Fur (ferric uptake regulation) genes have a similar function ${ }^{3}$. To acquire iron from host proteins, Neisseria species, Staphylococcus aureus, Streptococcus mutans, L. monocytogenes and Haemophilus influenzae express surface receptors, which interact with mammalian iron-binding proteins, such as TF and LF (REF. 3). To access and use host iron, bacteria, including E. coli and Vibrio cholerae, express haemo- or cytolysins, which release iron from intracellular iron complexes ${ }^{3}$. C. diphtheriae uses a haem-oxygenase to degrade haem and to access iron. S. mutans and L. monocytogenes express membranebound or secreted iron reductases to liberate iron from LF and TF (REF. 3). A recent study investigated the use of iron by S. aureus and showed that, during infection, these Gram-positive bacteria preferentially use haem-bound iron compared with TF-bound iron. In support of this finding, a gene cluster encoding a haem-transport system (HTS) that imports haem into staphylococci has recently been identified ${ }^{28}$. This strategy allows microbial access to $80 \%$ of the host's iron pool, a source of iron that far exceeds the $1 \%$ of the iron pool that is complexed with TF.

Intracellular growth of mycobacteria, Chlamydia species and Legionella pneumophila crucially depends on host-derived iron. Blocking iron uptake by infected cells inhibits the growth of these pathogens ${ }^{29-33}$. To access host-derived iron sources, these bacteria exploit specific intracellular niches in the host cell. M. tuberculosis, as well as other pathogenic mycobacteria, including Mycobacterium avium, block maturation of phagosomes, thereby maintaining this intracellular niche at an early endosomal stage (FIG. 1). In the early phagosome, mycobacteria can exploit the host cell's iron uptake system - the TF-TFR system ${ }^{34}$ (FIG. 3). When mycobacteriainfected macrophages were exposed to TF that was loaded with radioactive iron, these microorganisms incorporated the radioactive iron from TF (D. Russell, personal communication). Currently, it is unclear how iron is released from TF into the phagosome, thereby allowing mycobacterial uptake. It is also not known whether mycobacterial products are directly involved in this process, although it is possible that, owing to their higher affinity, mycobactins might compete with TF for iron. It has also been suggested that M. tuberculosis can directly access intracellular iron sources from the hostcell cytoplasm ${ }^{35}$. Iron has an important regulatory role in the virulence of L. monocytogenes. Important virulence factors that are expressed by this pathogen to facilitate bacterial intracellular invasion and spreading (including ActA, LLO and the regulator PrfA) are positively controlled by iron limitation ${ }^{36}$. This indicates that, at least in part, the requirement for iron by this pathogen constituted a strong evolutionary impetus to adapt to the cytoplasm of host cells.

The main cytoplasmic iron source in host cells is saturated holoferritin. Indeed, holoferritin allows the growth of listeriae in iron-free minimal medium, which otherwise cannot support listerial growth (U. Schaible and colleagues, unpublished observations). To provide intracytoplasmic listeriae with an easily accessible iron source, the listeria TF-like siderophore must have a higher affinity for iron than ferritin - a proposal that awaits experimental verification. L. monocytogenes expresses a surface-associated ferric reductase that can extract iron from ferritin, LF and TF (REF. 37). So far, the only microorganism that has been shown experimentally to exploit holoferritin as an iron source is Neisseria meningitidis ${ }^{38}$. In epithelial cells, intracellular meningococci recruit and aggregate ferritin. The aggregated molecule is rapidly degraded and free iron is released. Ferritin has not been implicated as a preferential iron source for other pathogens, probably because of its cytoplasmic location and the rapid removal of the molecule following cell damage. spanning transporter protein family that contains an ATP-binding cassette. 


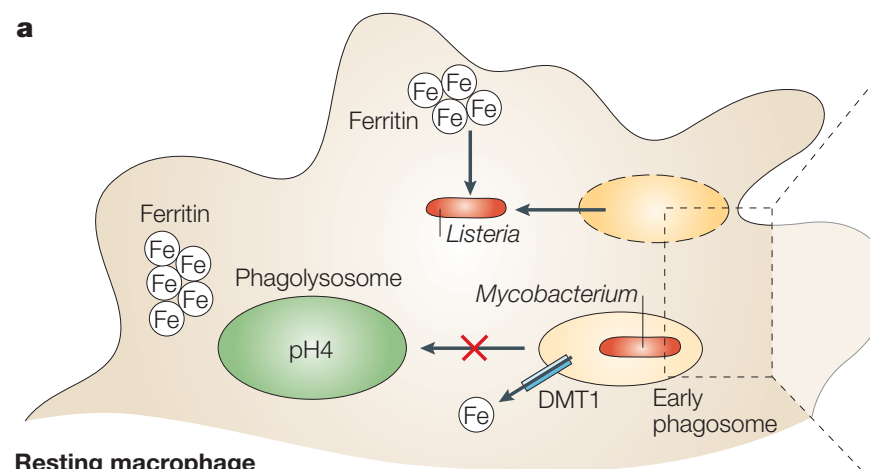

Resting macrophage
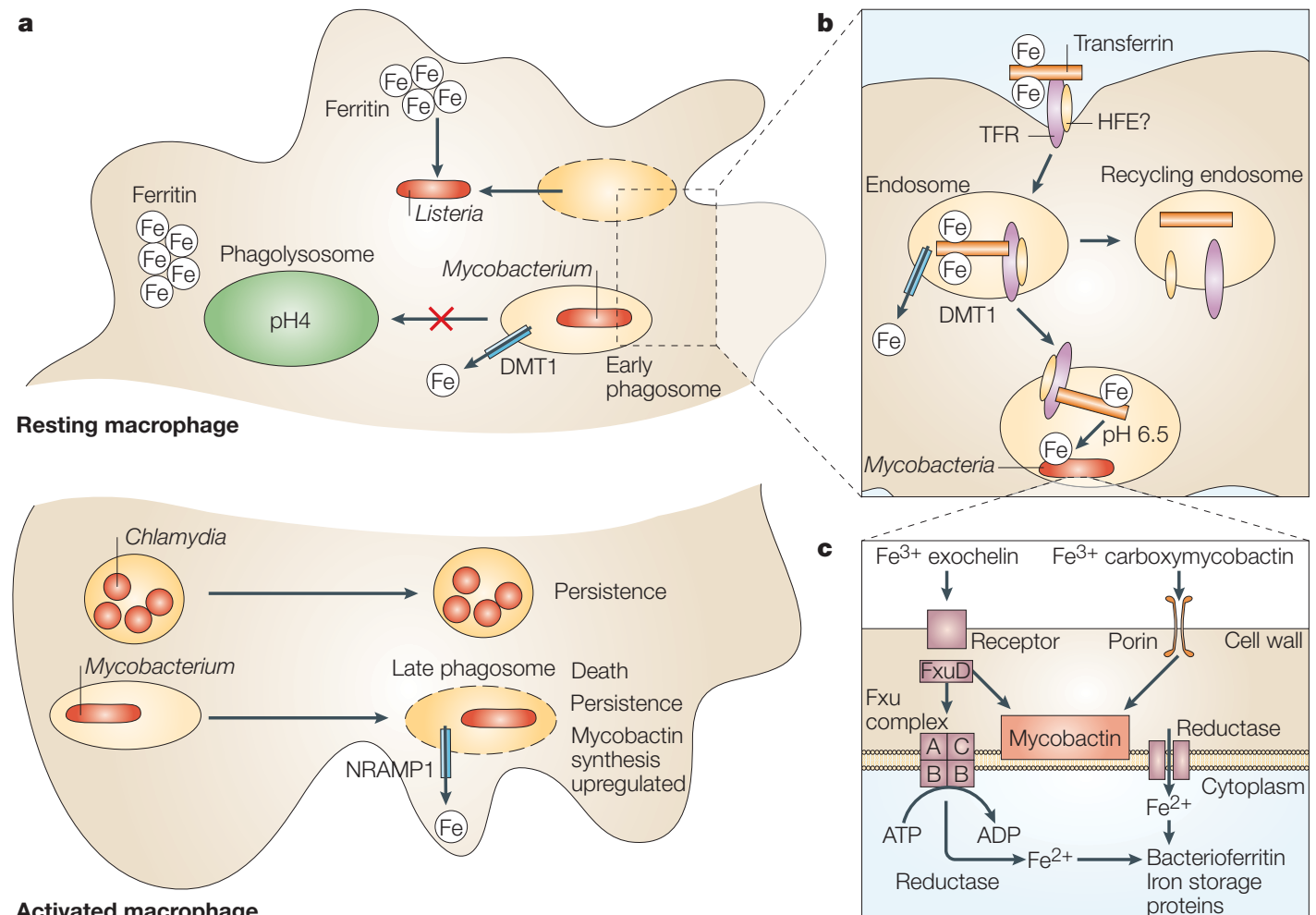

Activated macrophage

Figure 3 | Iron metabolism and infection. a | Listeriae, mycobacteria and chlamydiae depend on iron sources derived from the host. Listeriae might access cytoplasmic iron from ferritin (see text). Macrophage activation or iron depletion inhibits the growth of both mycobacteria and chlamydiae, leading to bacterial persistence. In response, mycobacteria express at least three iron uptake systems - exochelins, carboxymycobactins and mycobactins - which are upregulated in mycobacteria that are grown under conditions of iron stress. $\mathbf{b}$ | The iron-saturated form of transferrin, holotransferrin, binds to the transferrin receptor (TFR) on the surface of the macrophage. The haemochromatosis protein HFE might participate in this process. This complex delivers iron to the early endosome where divalent-metal transporter-1 (DMT1) facilitates the release of iron into the cytoplasm. Apoferritin and the TFR are recycled to the cell surface. In the early endosome, mycobacteria interact with the TF-TFR iron uptake system and exploit host-cell iron. c $\mid$ A complex of ferric iron $\left(\mathrm{Fe}^{3+}\right)$ and the mycobacterial siderophore exochelin is bound by a cell-wall receptor, after which iron is transported into the cytoplasm by the ATP-dependent Fxu complex (composed of FxuA, B and C). Another mycobacterial siderophore, carboxymycobactin, binds to $\mathrm{Fe}^{3+}$ and enters the cell wall through a porin. A periplasmic iron-binding protein, $\mathrm{FxuD}$, also promotes entry of free iron into the cell. Transport through the cell membrane is accompanied by the reduction of $\mathrm{Fe}^{3+}$ to ferrous iron $\left(\mathrm{Fe}^{2+}\right)$, which is subsequently incorporated into mycobacterial iron-containing proteins, including the bactoferitins. Excess iron is bound by the membrane-associated siderophore mycobactin. NRAMP1, natural resistance-associated macrophage protein-1.

In activated macrophages, mycobacteria can no longer block phagosome maturation and are secluded from the TF-TFR iron uptake system ${ }^{34,39}$. Furthermore, interferon- $\gamma$ (IFN $\gamma$ ) activation of macrophages downregulates TFR surface expression, probably to limit iron uptake $^{32,40}$. Consequently, mycobacterial growth is restricted under these conditions. A recent TRANSCRIPTOME ANALYSIS showed important differences in the expression of genes that were involved in iron metabolism in M. tuberculosis that was isolated from resting macrophages compared with activated macrophages. (H. Rachman and colleagues, unpublished observations). The expression of genes required for mycobactin synthesis $(m t b A$ and $m t b B)$ was increased in bacteria that were extracted from activated macrophages, whereas the expression of genes that encode the iron-storage proteins bacterioferritins $(b f r A)$ and iron-requiring enzymes, such as oxidases, was decreased, probably owing to the release of further iron for metabolic needs. Similarly, M. tuberculosis that was isolated from mouse lungs
4 to 9 weeks post-infection expressed $m t b B$ at high levels and $b f r A$ at low levels when compared with the gene expression signature of mycobacteria that were grown in vitro ${ }^{41}$. As discussed above, the regulation of these genes in M. tuberculosis involves the essential control element IdeR. Hierarchical clustering of all transcriptome data revealed a high similarity between the global gene expression patterns of $M$. tuberculosis from activated macrophages and those from mycobacteria grown in iron-depleted broth compared with gene expression profiles from bacteria grown in resting macrophages or in iron-supplemented broth (H. Rachman and colleagues, unpublished observations). These data indicate that, in activated macrophages, mycobacteria suffer from iron stress, indicating that the global gene expression response is strongly influenced by iron limitation. Downregulation of TFR expression in IFN $\gamma$-activated macrophages probably also limits ROI production, thereby avoiding inflammatory pathological sequelae. 
Iron overload in the host, owing to nutritional, hereditary or therapeutic reasons, exacerbates several infectious diseases, including yersiniosis, salmonellosis, tuberculosis and AIDS. Patients with hereditary tissue iron overload - known as haemochromatosis - caused by mutations in the HFE gene have an increased incidence of salmonellosis and yersiniosis ${ }^{9,42}$. Clinical studies of prophylactic iron supplementation to treat anaemia also showed a direct correlation between iron uptake and an increased risk of these infections, as well as tuberculosis ${ }^{43,44}$. Iron supplementation has been recognized as a risk factor for tuberculosis since the nineteenth century. In his Lectures in Clinical Medicine, Armand Trousseau described an anaemic girl, with no obvious clinical signs of infection, who rapidly succumbed to reactivated tuberculosis after commencing iron supplementation ${ }^{45}$. Recent epidemiological studies in African societies indicate that nutritional iron overload from high iron uptake, either from crops that are grown on volcanic soil or from traditional beer that is brewed in iron pots from local grains, is a risk factor for increased morbidity and mortality from active tuberculosis ${ }^{46-48}$. There is also a link between dietary iron overload and a nonHLA-based genetic predisposition - probably the recently described mutations in the ferroportin-1 gene — to tissue iron overload in certain African populations $^{49}$. Similarly, in experimental tuberculosis in mice, the mycobacterial burden was not only elevated under dietary iron-overload conditions, but also in $\beta 2 \mathrm{~m}$-knockout mice that have a non-functional HFE protein with a resultant hereditary iron-overload phenotype ${ }^{50}$. It should be pointed out, however, that haemochromatosis has not been identified as a risk factor for human tuberculosis. The $\beta 2 \mathrm{~m}$-knockout mice have several other immune-response deficiencies; for example, these mice do not express MHC class I and CD1d molecules and, consequently, lack CD8 ${ }^{+}$ and NATURAL KILLER (NK) T CELLS. However, knockout mice with the MHC class I and CD1d mutant phenotype are less susceptible to tuberculosis than $\beta 2 \mathrm{~m}$-knockout mice $^{51,52}$. Of note, iron sequestration in $\beta 2 \mathrm{~m}$-knockout mice reduced the elevated mycobacterial burden to levels seen in mice lacking MHC class-I molecules ${ }^{32}$. Therefore, the increased susceptibility of $\beta 2 \mathrm{~m}$-knockout mice to mycobacterial infection is far more complex than originally envisaged, comprising both iron overload and different T-cell deficiencies.

A small subset of $\mathrm{T}$ cells that express markers of both NK cells and $\mathrm{T}$ cells. They express a T-cell receptor of limited diversity and are restricted by CD1d.

\section{BIOFILM}

A dense layer of bacteria in which the bacterial cells are enclosed by an extracellular matrix.

CYSTIC FIBROSIS An autosomal recessive disease characterized by pulmonary pathology and dysfunction of exocrine glands. might downregulate NO production ${ }^{53}$. By contrast, depleting mice of iron using deferroxamine exacerbated S. typhimurium infection through the inhibition of ROI production ${ }^{17}$. Similarly, iron depletion of listeriainfected macrophages blocked ROI production and enhanced listerial growth ${ }^{54}$. These data emphasize that the respiratory burst is a vital defence mechanism against salmonellae, listeriae and other bacteria.

Lactoferrin is a high-affinity iron-binding protein that is present in body fluids such as milk, saliva, tears and serum, and is released from neutrophils on degranulation ${ }^{55,56}$. In circulation, LF is incompletely saturated with iron and therefore it can clear free iron from the tissues. Consequently, LF functions as a firstline defence molecule against invading microorganisms through its ability to sequester iron. Iron sequestration by exogenous LF corrected the exacerbation of tuberculosis in $\beta 2 \mathrm{~m}$-knockout mice ${ }^{32}$. The iron-sequestering function of LF has also been described as a vital, innate defence mechanism against Pseudomonas aeruginos $a^{57}$. LF inhibits BIOFILM formation in patients with CYSTIC FIBROsIs, and this mechanism provides a rationale for the development of therapeutic strategies to counteract $P$. aeruginosa biofilm formation in these patients. Feeding mice with LF also decreases the endotoxin burden in the intestine, indicating a lower degree of intestinal settlement ${ }^{58}$. Furthermore, the LF sequence comprises a defensin-like peptide, lactoferricin, which shows microbicidal activity against Candida albicans, S. mutans, V. cholerae and various enterobacteria ${ }^{55,56,59-62}$. Although these data indicate that LF has direct anti-infective functions, LF-knockout mice do not show heightened susceptibility to various pathogens, most notably M. tuberculosis (P. Ward and U. Schaible, unpublished observations). Therefore, the antimicrobial functions of LF deserve further analysis, especially in the context of the newborn host. The beneficial effects of breast feeding and of milk in general — although mostly anecdotal — may at least partially be due to LF.

Apart from direct effects on microbial replication and innate immunity, iron availability also influences acquired immune responses ${ }^{63}$. Iron deficiency downregulates $\mathrm{T}$-cell responses in several experimental models. T cells from iron-depleted mice show reduced antigen-specific as well as polyclonal proliferation, and an important co-stimulatory receptor that functions in T-cell activation, CD28, is downregulated under these conditions ${ }^{64}$. CD $4{ }^{+} \mathrm{T}$-cell-driven experimental allergic encephalitis, which is used as a mouse model of multiple sclerosis, is absent in iron-deficient mice ${ }^{65}$. In a comparative study, iron-overloaded mice showed reduced contact-mediated sensitivity reactions as well as lower IFN $\gamma$ production and IgM secretion ${ }^{66,67}$. Surprisingly, in iron-deficient mice, a similar outcome was observed. These mice had a more generalized immune deficiency that encompassed reduced delayedtype hypersensitivity (DTH), reduced contact-mediated sensitivity, impaired T-cell activation in vitro and lowered IgM and IgG serum levels ${ }^{66,67}$. Both elevated and reduced iron availability impaired host immune 
CYTOKINES

Biologically active molecules that are released by cells (mainly leukocytes) and that modulate the function of other cells by binding to specific receptors. responses, which impressively illustrates the importance of a normal iron balance for optimal functioning of the host immune system. Similarly, patients with iron overload caused by either thalassaemia or haemochromatosis frequently have reduced $\mathrm{CD}^{+} \mathrm{T}$-cell counts, which respond to treatment with iron chelators, such as deferroxamine ${ }^{68,69} . \beta$-thalassaemic patients have a higher risk of infections with various pathogens including Salmonella enterica, Klebsiella pneumoniae, Streptococcus pneumoniae, Burkholderia pseudomallei and the fungus Pythidium insidiosum ${ }^{70}$. Iron overload also modulates L. major infection in mice and results in a lower parasite burden as well as reduced interleukin (IL)-4 and IL-10 production and increased IFN $\gamma$ and inducible nitric oxide synthase (iNOS) expression ${ }^{71}$. In patients with iron overload as measured by TF saturation, higher levels of the antiinflammatory CYTOKINEs IL-4 and IL-10, as well as IL-6, were observed. By contrast, iNOS expression was blocked $^{72}$. There is also an interesting link between these cytokines and iron regulation as IL- 6 induces hepcidin expression, thereby leading to hypoferraemia ${ }^{73}$. This might explain the anaemia of inflammation (also known as anaemia of chronic disease), which occurs in patients with chronic infections and inflammation, probably as a mechanism to limit the iron that is available to pathogens. Taken together, these data indicate that the higher susceptibility to infection of the iron-overloaded host is not only owing to improved access of the pathogen to this essential element, but is also a consequence of the direct impairment of innate and acquired immune responses.

\section{Conclusion}

If the importance of iron in microbial infections of global importance such as tuberculosis is accepted, it is tempting to speculate that new intervention strategies can be developed that capitalize on our knowledge of the iron equilibrium between the host and the infecting microorganism. Such a strategy would aim to tip the balance in favour of the host through restricting the access of pathogens to iron and, at the same time, maintaining sufficient levels of iron to allow the host to instigate iron-dependent microbicidal effector mechanisms. This goal could be achieved either by specifically blocking iron-uptake systems of the pathogen with antibiotics, or by reducing the availability of iron in the intracellular niche occupied by the microorganism. Finally, the therapeutic potential of iron-sequestering molecules such as LF should be investigated in more detail and exploited to their full potential. Indeed, this general approach could be applied to a diverse range of essential nutrients for which the host and pathogen have a shared requirement, thereby allowing the development of new nutrition-based intervention strategies to support more established prophylactic and therapeutic approaches to conquer infectious disease.
1. Hentze, M. W., Muckenthaler, M. U. \& Andrews, N. C. Balancing acts: molecular control of mammalian iron metabolism. Cell 117, 285-297 (2004).

A comprehensive review of our current understanding of mammalian iron metabolism.

2. Kaplan, J. Mechanisms of cellular iron acquisition: another iron in the fire. Cell 111, 603-606 (2002).

3. Andrews, S. C., Robinson, A. K. \& Rodriguez-Quinones, F. Bacterial iron homeostasis. FEMS Microbiol. Rev. 27, 215-237 (2003).

4. Picard, V., Govoni, G., Jabado, N. \& Gros, P. Nramp 2 (DCT1/DMT1) expressed at the plasma membrane transports iron and other divalent cations into a calcein-accessible cytoplasmic pool. J. Biol. Chem. 275 35738-35745 (2000)

5. Donovan, A. et al. Positional cloning of zebrafish ferroportin identifies a conserved vertebrate iron exporter. Nature $\mathbf{4 0 3}$ 776-781 (2000).

6. Ganz, T. Hepcidin, a key regulator of iron metabolism and mediator of anemia of inflammation. Blood 102, 783-788 (2003).

7. Gruenheid, S. et al. The iron transport protein NRAMP2 is an integral membrane glycoprotein that colocalizes with transferrin in recycling endosomes. J. Exp. Med. 189, 831-841 (1999).

8. Robson, K. J. et al. Recent advances in understanding haemochromatosis: a transition state. J. Med. Genet. 41, 721-730 (2004).

9. Pietrangelo, A. Hereditary hemochromatosis-a new look at an old disease. N. Engl. J. Med. 350, 2383-2397 (2004).

10. Eisenbach, C., Gehrke, S. G. \& Stremmel, W. Iron, the HFE gene, and hepatitis C. Clin. Liver Dis. 8, 775-785 (2004).

11. Bennett, M. J., Lebron, J. A. \& Bjorkman, P. J. Crystal structure of the hereditary haemochromatosis protein HFE complexed with transferrin receptor. Nature 403, 46-53 (2000).

12. Enns, C. A. Pumping iron: the strange partnership of the hemochromatosis protein, a class I MHC homolog, with the transferrin receptor. Traffic 2, 167-174 (2001).

13. Nicolas, G. et al. Constitutive hepcidin expression prevents iron overload in a mouse model of hemochromatosis. Nature Genet. 34, 97-101 (2003).

This paper describes the essential function of hepcidin in iron metabolism.
14. Canonne-Hergaux, F., Gruenheid, S., Govoni, G. \& Gros, P. The Nramp1 protein and its role in resistance to infection and macrophage function. Proc. Assoc. Am. Physicians 111, 283-289 (1999).

15. Hackam, D J. et al. Host resistance to intracellular infection: mutation of natural resistance-associated macrophage protein 1 (Nramp1) impairs phagosomal acidification. J. Exp. Med. 188, 351-364 (1998).

16. Jabado, N., Cuellar-Mata, P., Grinstein, S. \& Gros, P. Iron chelators modulate the fusogenic properties of Salmonella-containing phagosomes. Proc. Natl Acad. Sci. USA 100, 6127-6132 (2003).

17. Collins, H. L., Kaufmann, S. H. E. \& Schaible, U. E. Iron chelation via deferoxamine exacerbates experimenta salmonellosis via inhibition of the nicotinamide adenine dinucleotide phosphate oxidase-dependent respiratory burst. J. Immunol. 168, 3458-3463 (2002).

18. Mogues, T., Goodrich, M. E., Ryan, L., LaCourse, R. \& North, R. J. The relative importance of T cell subsets in immunity and immunopathology of airborne Mycobacterium tuberculosis infection in mice. J. Exp. Med. 193, 271-80 (2001)

19. Posey, J. E. \& Gherardini, F. C. Lack of a role for iron in the Lyme disease pathogen. Science 288, 1651-1653 (2000).

This work describes the unusual case of a bacterial pathogen, which does not require iron.

20. Weinberg, E. D. Iron and infection. Microbiol. Rev. 42, 45-66 (1978)

21. Ratledge, C. \& Dover, L. G. Iron metabolism in pathogenic bacteria. Annu. Rev. Microbiol. 54, 881-941 (2000). A comprehensive review on our current knowledge of bacterial iron uptake systems.

22. Ratledge, C. Iron, mycobacteria and tuberculosis. Tuberculosis (Edinb.) 84, 110-130 (2004).

23. Brown, J. S. \& Holden, D. W. Iron acquisition by Gram-positive bacterial pathogens. Microbes Infect. 4, 1149-1156 (2002).

24. Agranoff, D. D. \& Krishna, S. Metal ion homeostasis and intracellular parasitism. Mol. Microbiol. 28, 403-412 (1998).

25. Rodriguez, G. M. \& Smith, I. Mechanisms of iron regulation in mycobacteria: role in physiology and virulence. Mol. Microbiol. 47, 1485-1494 (2003).
26. Manabe, Y. C., Saviola, B. J., Sun, L., Murphy, J. R. \& Bishai, W. R. Attenuation of virulence in Mycobacterium tuberculosis expressing a constitutively active iron repressor. Proc. Natl Acad. Sci. USA 96, 12844-12848 (1999).

27. Gold, B., Rodriguez, G. M., Marras, S. A., Pentecost, M. \& Smith, I. The Mycobacterium tuberculosis IdeR is a dual functional regulator that controls transcription of genes involved in iron acquisition, iron storage and survival in macrophages. Mol. Microbiol. 42, 851-865 (2001).

28. Skaar, E. P., Humayun, M., Bae, T., DeBord, K. L. \& Schneewind, O. Iron-source preference of Staphylococcus aureus infections. Science 305, 1626-1628 (2004). This paper demonstrates that haem-bound iron is the main iron source for staphylococci.

29. Al Younes, H. M., Rudel, T., Brinkmann, V., Szczepek, A. J. \& Meyer, T. F. Low iron availability modulates the course of Chlamydia pneumoniae infection. Cell. Microbiol. 3, 427-437 (2001)

30. Byrd, T. F. \& Horwitz, M. A. Lactoferrin inhibits or promotes Legionella pneumophila intracellular multiplication in nonactivated and interferon ã-activated human monocytes depending upon its degree of iron saturation. Iron-lactoferrin and nonphysiologic iron chelates reverse monocyte activation against Legionella pneumophila. J. Clin. Invest. 88, 1103-1112 (1991).

31. Olakanmi, O., Britigan, B. E. \& Schlesinger, L. S. Gallium disrupts iron metabolism of mycobacteria residing within human macrophages. Infect. Immun. 68, 5619-5627 (2000).

32. Schaible, U. E., Collins, H. L., Priem, F. \& Kaufmann, S. H. E. Correction of the iron overload defect in $\beta$-2-microglobulin knockout mice by lactoferrin abolishes their increased susceptibility to tuberculosis. J. Exp. Med. 196, 1507-1513 (2002).

Shows that, in an animal model, iron overload accelerates tuberculosis and that lactoferrin can be used to correct this effect.

33. Lounis, N., Truffot-Pernot, C., Grosset, J., Gordeuk, V. R. \& Boelaert, J. R. Iron and Mycobacterium tuberculosis infection. J. Clin. Virol. 20, 123-126 (2001).

34. Schaible, U., Collins, H. \& Kaufmann, S. H. E. Confrontation between intracellular bacteria and the immune system. Adv. Immunol. 71, 267-377 (1999). 
35. Olakanmi, O., Schlesinger, L. S., Ahmed, A. \& Britigan, B. E. Intraphagosomal Mycobacterium tuberculosis acquires iron from both extracellular transferrin and intracellular iron pools. Impact of interferon-ã and hemochromatosis. J. Biol. Chem. 277, 49727-49734 (2002)

36. Bockmann, R., Dickneite, C., Middendorf, B., Goebel, W. \& Sokolovic, Z. Specific binding of the Listeria monocytogenes transcriptional regulator PrfA to target sequences requires additional factor(s) and is influenced by iron. Mol. Microbiol. 22, 643-653 (1996).

37. Deneer, H. G, Healey, V. \& Boychuk, I. Reduction of exogenous ferric iron by a surface-associated ferric reductase of Listeria spp. Microbiology 141, 1985-1992 (1995).

38. Larson, J. A., Howie, H. L. \& So, M. Neisseria meningitidis accelerates ferritin degradation in host epithelial cells to yield an essential iron source. Mol. Microbiol. 53, 807-820 (2004).

This paper identifies meningococci as the first pathogens to use ferritin as a host-derived iron source.

39. Schaible, U. E., Sturgill-Koszycki, S., Schlesinger, P. H. \& Russell, D. G. Cytokine activation leads to acidification and increases maturation of Mycobacterium avium-containing phagosomes in murine macrophages. J. Immunol. 160, 1290-1296 (1998)

40. Byrd, T. F. \& Horwitz, M. A. Regulation of transferrin recepto expression and ferritin content in human mononuclear phagocytes. Coordinate upregulation by iron transferrin and downregulation by interferon $\%$. J. Clin. Invest. 91, 969-976 (1993).

41. Timm, J. et al. Differential expression of iron-, carbon-, and oxygen-responsive mycobacterial genes in the lungs of chronically infected mice and tuberculosis patients. Proc. Natl Acad. Sci. USA 100, 14321-14326 (2003).

42. Moalem, S., Weinberg, E. D. \& Percy, M. E. Hemochromatosis and the enigma of misplaced iron: implications for infectious disease and survival. Biometals 17, 135-139 (2004)

43. Murray, M. J., Murray, A. B., Murray, M. B. \& Murray, C. J. The adverse effect of iron repletion on the course of certain infections. Br. Med. J. 2, 1113-1115 (1978).

44. Weinberg, E. D. Iron loading and disease surveillance. Emerg. Infect. Dis. 5, 346-352 (1999).

45. Trousseau, A. True and false chlorosis. Lectures on Clinical Medicine 5, 95-117 (1872).

46. Gordeuk, V. R. African iron overload. Semin. Hematol. 39 263-269 (2002).

47. Gangaidzo, I. T. et al. Association of pulmonary tuberculosis with increased dietary iron. J. Infect. Dis. 184, 936-939 (2001).

This article demonstrates the epidemiological correlation between iron overload and the risk of tuberculosis in a human population.

48. Gordeuk, V. R., McLaren, C. E., MacPhail, A. P., Deichsel, G. \& Bothwell, T. H. Associations of iron overload in Africa with hepatocellular carcinoma and tuberculosis: Strachan's 1929 thesis revisited. Blood 87, 3470-3476 (1996).
49. Gordeuk, V. et al. Iron overload in Africa. Interaction between a gene and dietary iron content. N. Engl. J. Med. $\mathbf{3 2 6}$ 95-100 (1992)

50. Santos, M., Clevers, H., De Sousa, M. \& Marx, J. J. Adaptive response of iron absorption to anemia, increased erythropoiesis, iron deficiency, and iron loading in $\beta 2$ microglobulin knockout mice. Blood 91, 3059-3065 (1998).

51. Rolph, M. S. et al. MHC class la-restricted T cells partially account for $\beta 2$-microglobulin-dependent resistance to Mycobacterium tuberculosis. Eur. J. Immunol. 31 1944-1949 (2001).

52. Behar, S. M., Dascher, C. C., Grusby, M. J., Wang, C. R. \& Brenner, M. B. Susceptibility of mice deficient in CD1D or TAP1 to infection with Mycobacterium tuberculosis. J. Exp. Med. 189, 1973-1980 (1999).

53. Bartfay, W. J. \& Bartfay, E. Systemic oxygen-free radical production in iron-loaded mice. West. J. Nurs. Res. 22, 927-935 (2000).

54. Alford, C. E., King, T. E. Jr \& Campbell, P. A. Role of transferrin, transferrin receptors, and iron in macrophage listericidal activity. J. Exp. Med. 174, 459-466 (1991).

55. Ward, P. P., Uribe-Luna, S. \& Conneely, O. M. Lactoferrin and host defense. Biochem. Cell Biol. 80, 95-102 (2002).

56. Vorland, L. H. Lactoferrin: a multifunctional glycoprotein. APMIS 107, 971-981 (1999).

57. Singh, P. K., Parsek, M. R., Greenberg, E. P. \& Welsh, M. J. A component of innate immunity prevents bacterial biofilm development. Nature 417, 552-555 (2002). This study reveals the importance of lactoferrin in innate immunity.

58. Griffiths, E. A. et al. In vivo effects of bifidobacteria and lactoferrin on gut endotoxin concentration and mucosal immunity in Balb/c mice. Dig. Dis. Sci. 49, 579-589 (2004).

59. Tanida, T., Rao, F., Hamada, T., Ueta, E. \& Osaki, T. Lactoferrin peptide increases the survival of Candida albicans-inoculated mice by upregulating neutrophil and macrophage functions, especially in combination with amphotericin B and granulocyte-macrophage colonystimulating factor. Infect. Immun. 69, 3883-3890 (2001).

60 Ueta, E., Tanida, T., \& Osaki, T. A novel bovine lactoferrin peptide, FKCRRWQWRM, suppresses Candida cell growth and activates neutrophils. J. Pept. Res. 57, 240-249 (2001).

61. Tanida, T., Rao, F., Hamada, T., Ueta, E. \& Osaki, T. Lactoferrin peptide increases the survival of Candida albicans-inoculated mice by upregulating neutrophil and macrophage functions, especially in combination with amphotericin B and granulocyte-macrophage colonystimulating factor. Infect. Immun. 69, 3883-3890 (2001).

62. Yamauchi, K., Tomita, M., Giehl, T. J. \& Ellison, R. T. Antibacterial activity of lactoferrin and a pepsin-derived lactoferrin peptide fragment. Infect. Immun. 61, 719-728 (1993).

63. Walker, E. M. Jr \& Walker, S. M. Effects of iron overload on the immune system. Ann. Clin. Lab. Sci. 30, 354-365 (2000).

64. Kuvibidila, S. R. \& Porretta, C. Iron deficiency and in vitro iron chelation reduce the expression of cluster of differentiation molecule (CD)28 but not CD3 receptors on murine thymocytes and spleen cells. Br. J. Nutr. 90 179-189 (2003)

65. Grant, S. M., Wiesinger, J. A., Beard, J. L. \& Cantorna, M. T. Iron-deficient mice fail to develop autoimmune encephalomyelitis. J. Nutr. 133, 2635-2638 (2003).

66. Omara, F. O. \& Blakley, B. R. The IgM and IgG antibody responses in iron-deficient and iron-loaded mice. Biol. Trace Elem. Res. 46, 155-161 (1994).

67. Omara, F. O. \& Blakley, B. R. The effects of iron deficiency and iron overload on cell-mediated immunity in the mouse. Br. J. Nutr. 72, 899-909 (1994).

68. Cunningham-Rundles, S. et al. Effect of transfusional iron overload on immune response. J. Infect. Dis. 182 S115-S121 (2000).

69. Wanachiwanawin, W. Infections in $\varepsilon-\beta$-thalassemia J. Pediatr. Hematol. Oncol. 22, 581-587 (2000).

70. de Sousa, M. \& Porto, G. The immunological system in hemochromatosis. J. Hepatol. 28, (Suppl. 1) 1-7 (1998).

71. Bisti, S. et al. The outcome of Leishmania major experimental infection in BALB/c mice can be modulated by exogenously delivered iron. Eur. J. Immunol. 30, 3732-3740 (2000).

72. Weiss, G. et al. Associations between cellular immune effector function, iron metabolism, and disease activity in patients with chronic hepatitis $\mathrm{C}$ virus infection. J. Infect. Dis. 180, 1452-1458 (1999).

73. Nemeth, E. et al. IL-6 mediates hypoferremia of inflammation by inducing the synthesis of the iron regulatory hormone hepcidin. J. Clin. Invest. 113 1271-1276 (2004)

This paper elucidates an important link between innate immunity and regulation of iron metabolism.

Acknowledgements

Financial support was provided to S.H.E.K and U.E.S. by the Deutsche Forschungsgemeinschaft. The authors thank L. Fehlig for wonderful help with the figures and J. Koth for expert secretarial assistance.

Competing interests statement

The authors declare no competing financial interests.

\section{(2) Online links}

\section{DATABASES}

The following terms in this article are linked online to:

Entrez: http://www.ncbi.nlm.nih.gov/Entrez/

bfrA | ferroportin-1 | HFE | Nramp1

Interpro: http://www.ebi.ac.uk/interpro/

CD163 | DCYTB | DMT1 | FepA | FepB | ferroportin | IdeR | TFR2

TonB

\section{FURTHER INFORMATION}

Stefan H. E. Kaufmann's laboratory:

www.mpiib-berlin.mpg.de

Access to this links box is available online. 


\section{REV I E W S}

CORRECTION

\section{IRON AND MICROBIAL INFECTION}

Ulrich E. Schaible and Stefan H. E. Kaufmann

Nature Rev. Microbiol. 2, 946-953 (2004)

The citation given to support the statement that the solubility of $\mathrm{Fe}(\mathrm{OH})_{2}{ }^{+}$at $\mathrm{pH} 7$ is $1.4 \times 10^{-9}$ was incorrect, and should be:

3. Chipperfield, J. R. \& Ratledge, C. Salicylic acid is not a bacterial siderophore: a theoretical study. Biometals 13, 165-168 (2000).

The online version of this article has been corrected. 